Original Article

\title{
Intra-rater reliability of arch height ratio measurement using the navicular tuberosity on the surface of the body in children with Down syndrome
}

\author{
Yoshinide Kanai, RPT, PhD ${ }^{1)^{*}}$, Hirotaka Mutsuzaki, MD, PhD ${ }^{2)}$, TAKumi Komuro, RPT ${ }^{3)}$ \\ 1) Department of Physical Therapy, Ibaraki Prefectural University of Health Sciences: \\ 4669-2 Ami-machi Ami, Inashiki-gun, Ibaraki 300-0394, Japan \\ 2) Department of Orthopaedic Surgery, Ibaraki Prefectural University of Health Sciences, Japan \\ 3) Department of Physical Therapy, Shimura Omiya Hospital, Japan
}

\begin{abstract}
Purpose] Flat feet frequently present in children with Down syndrome, necessitating a clinical evaluation and diagnosis. Therefore, a simple, low cost diagnostic method that can avoid radiation exposure is needed. This study was performed to investigate the intra-rater reliability of arch height ratio measurement using bone markers of the foot on the surface of the body in children with Down syndrome. [Participants and Methods] In total, 27 feet of 27 children with Down syndrome (16 male, 11 female) were included. We measured the length of each foot and the height of the navicular tuberosity on the surface of the body. The arch height ratio was calculated using the length and height. The same examiner performed three consecutive measurements of each of the two parameters. We calculated the arch height ratio using each of these measurements. The intraclass correlation coefficient $[\operatorname{ICC}(1,3)]$ of the length of the foot, height of the navicular tuberosity, and arch height ratio among the three measurements was obtained. [Results] The ICC of the height of the navicular tuberosity, length of the foot, and arch height ratio were quite high at $0.998,0.999$, and 0.997 , respectively. [Conclusion] This study showed high intra-rater reliability of arch height ratio measurement using the length of the foot and height of the navicular tuberosity on the surface of the body in children with Down syndrome.

Key words: Down syndrome, Arch height ratio, Reliability
\end{abstract}

(This article was submitted Dec. 14, 2018, and was accepted Feb. 19, 2019)

\section{INTRODUCTION}

A previous study showed that $88 \%$ of patients with Down syndrome (DS) had flat feet in an institution ${ }^{1)}$. In an experimental study in which the feet were aligned to imitate flat feet (the foot portion was excessively pronated), a significant increase in malalignment as indicated by pelvic anteversion and internal shank rotation was confirmed ${ }^{2}$. Moreover, the medial longitudinal arch also plays a role in shock absorption and load support ${ }^{3}$.

Radiographs are used to diagnose flat feet. However, excessive radiation exposure and high costs associated with repeated follow-up examinations should ideally be avoided. Thus, a new and easier way to assess the condition of the feet in patients with DS with neither radioactive exposure nor high financial costs is needed.

The arch height ratio is an index used to objectively assess the medial arch of the sole without the use of X-rays ${ }^{4}$. Arch measurement (navicular bone height $\times 100$ / foot length) using markers on the surface of the body can indicate the condition of the medial longitudinal $\mathrm{arch}^{4}$. However, no reports on measurement of the arch height ratio have performed the measure-

*Corresponding author. Yoshihide Kanai (E-mail: kanai@ipu.ac.jp)

(C2019 The Society of Physical Therapy Science. Published by IPEC Inc.

(c) (1) $\odot$ This is an open-access article distributed under the terms of the Creative Commons Attribution Non-Commercial No Derivatives

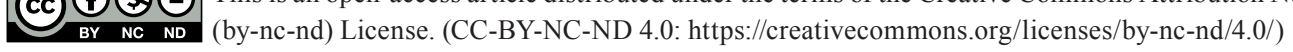


Table 1. Mean age and arch height ratio in children with Down syndrome

\begin{tabular}{lcc}
\hline & Age (years) & Arch ratio $(\%)$ \\
\hline Male $(\mathrm{n}=16)$ & $12.5 \pm 3.8$ & $14.8 \pm 4.1$ \\
Female $(\mathrm{n}=11)$ & $13.4 \pm 2.1$ & $13.5 \pm 2.4$ \\
\hline
\end{tabular}

Data are presented as mean \pm standard deviation.

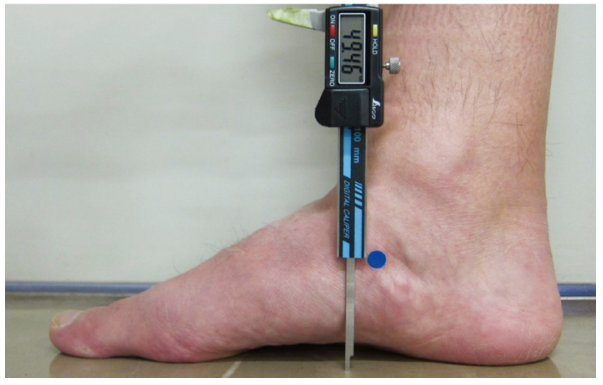

Fig. 1. Measurement of the height of the navicular bone from the body surface.

ment on the body surface using the navicular tuberosity as an indicator in patients with DS. These patients cannot always be evaluated with good repeatability because of their limited ability to understand instructions and the flaccidity of their soft tissue. Therefore, it is necessary to first evaluate whether arch height ratio measurement is reproducible using bone markers on the body surface to determine the reliability of this technique.

The purpose of this study was to investigate the intra-rater reliability of arch height ratio measurement using bone markers of the foot on the surface of the body in children with DS. Using this method, the diagnosis and follow-up of flat feet in patients with DS may be facilitated without using radiographs.

\section{PARTICIPANTS AND METHODS}

The participants in this study were recruited from a special-needs school for children with intellectual disability. Twentyseven participants (16 male, 11 female) were included (Table 1). The inclusion criteria were the presence of DS, the ability to understand simple instructions given by an examiner, and the ability to stand without assistance. The feet to be measured were arbitrarily set on the right side in all participants.

The procedure for measuring the arch height ratio was as follows. The participant was instructed to stand still with the feet shoulder-width apart. The researcher modified the posture to ensure that the participant stood symmetrically. The highest point of the navicular tuberosity that was palpable from the medial side was defined as the height of the navicular tuberosity. The navicular tuberosity was easily detected as a convex structure resembling a small spur. A seal was placed on the skin to mark the point of the navicular tuberosity. The length of the foot and height of the navicular tuberosity were measured between ground and the tuberosity using two sizes of digital calipers (150 mm and $300 \mathrm{~mm}$ ) (Shinwa Rules Co., Ltd., Niigata, Japan). We obtained the height of navicular tuberosity as shown in Fig. 1. Each parameter was measured by one examiner. The arch height ratio was calculated using the length and height. The same examiner performed three consecutive measurements of each parameter according to the same seal that had been placed as a marker. After the height or length had been measured using the device, the examiner released it from the targeted point and then immediately placed the device on the surface of body and measured the parameter again. This procedure was repeated three times.

The intraclass correlation coefficient $[\operatorname{ICC}(1,3)]^{5)}$ of each value (length of the foot, height of the navicular tuberosity, and arch height ratio) among the three measurements was obtained. Statistical analyses were performed using SPSS Statistics ver. 24 (IBM Corp., Armonk, NY, USA). The level of statistical significance was set at $\mathrm{p}=0.05$.

This study was approved by the Ibaraki Prefectural University of Health Sciences Ethics Committee (approval no. 779). All participants and their parents received a thorough explanation with documents, and written informed consent from the children's parents was obtained.

\section{RESULTS}

The ICCs of the three parameters are shown in Table 2. The ICCs of the height of the navicular tuberosity, length of the foot, and arch height ratio were quite high at $0.998,0.999$, and 0.997 , respectively.

\section{DISCUSSION}

The high ICCs in the present study indicated good intra-rater reliability among the three measurements. This result suggests that measurement of the arch height ratio on the surface of the body by the same examiner is a reliable method of evaluating foot conditions in people with DS. Using this method, medical professionals such as orthopaedic doctors, physical therapists, and orthotists may be able to effectively evaluate the foot condition, especially the presence of flat feet, in patients with DS. We measured the foot length from the end of the calcaneus to the tip of the toe located at the front-most part on the 
Table 2. Foot parameters in children with Down syndrome

\begin{tabular}{|c|c|c|c|c|c|}
\hline \multirow{2}{*}{ Parameter } & \multicolumn{3}{|c|}{ Mean } & \multirow{2}{*}{$\mathrm{ICC}$} & \multirow{2}{*}{$\mathrm{p}$ value } \\
\hline & $1 \mathrm{st}$ & 2 nd & $3 \mathrm{rd}$ & & \\
\hline Height of navicular tuberosity (mm) & $28.7 \pm 8.0$ & $28.6 \pm 7.9$ & $28.8 \pm 8.0$ & 0.998 & $<0.001$ \\
\hline Arch height ratio $(\%)$ & $14.3 \pm 3.6$ & $14.3 \pm 3.6$ & $14.3 \pm 3.7$ & 0.997 & $<0.001$ \\
\hline
\end{tabular}

Data are presented as mean \pm standard deviation. ICC: intraclass correlation coefficient.

lateral view through the body surface. The foot measurements were simple because the points used as markers were clear. Because the arch height ratio is calculated from the foot length and navicular height, the measurement accuracy of the foot length/navicular height can be easily ascertained. Therefore, the reliability of the arch height ratio also increases. Although the inter-rater reliability of this technique has not been verified in people with DS, the navicular tuberosity was easily detected as a convex structure resembling a small spur in this study.

Meanwhile, because the measured values may change depending on the participant's standing posture and load condition, measuring a bone marker with the same posture is important. In our preparation for this study, we intended to identify an appropriate way to measure foot conditions. During preparation, we encountered inconsistency in the measured values. We found that this was due to the way the participant was standing. If the participant places more weight on one side, the posture will be asymmetrical. Individuals with DS have low muscle tonus, and the foot condition easily changes; this results in changes in the height of the navicular tuberosity. Thus, the standing position must be fixed during evaluation of foot conditions, and the posture must be symmetrical under instruction by an examiner. In the present study, we focused on a relatively comprehensive population of children with DS. However, it may be necessary to develop a body surface measurement technique that can be adapted to individuals with DS who have a lower intelligence quotient and are unable to understand the examiner's instructions.

This study has two main limitations. First, we did not compare this method with radiographs. Saltzman, et al. tried to find a best noninvasive method to evaluate the foot medial longitudinal arch through the correlation between the radiological index and the anthropometric one in 100 patients with foot problems ${ }^{6}$. As the result of this research, the correlation coefficient between an anthropometric parameter that the navicular height divided by foot length and a radiographic one that the talar height divided by foot length was 0.86 , and was very strong $\left.{ }^{6}\right)$. Other previous study reported the strongest correlation between a clinical measurement, normalized navicular height, and a radiological measure, calcaneal first metatarsal inclination angle $(\mathrm{r}=0.70)^{7}$. Normalized navicular height truncated is obtained by dividing the height of the navicular tuberosity from the supporting surface by the truncated foot length ${ }^{7}$. It also mentioned the strong association among the anthropometric and radiological parameters. However, the participants were not DS in the previous studies. Therefore, it is necessary to verify whether the scale measured from the body surface shows validity for flat feet in comparison with radiographic images regarding the arch height ratio measurement in patients with DS. Furthermore, not only intra-rater reliability within one examiner but also inter-rater reliability among different examiners needs to be verified despite the fact that the navicular tuberosity is easily detected. In patients with foot problems who were not DS, the inter-rater reliability of anthropometric measurement for foot has been already examined with ICC. The ICC between the examiners on the height of the navicular bone has been reported as 0.74 , which indicates that the reliability is $\operatorname{good}^{6}$ ). However, the research was also not implemented in patients with DS. Thus, the verification is required in population with DS. Further investigation is needed to determine whether this measurement technique is a more robust way of evaluating foot conditions in patients with DS.

In conclusion, we confirmed high repeatability within one examiner in the measurement of the arch height ratio using the length of the foot and the height of the navicular tuberosity on the surface of the body in children with DS.

\section{Funding}

This work was supported by Grant-in-Aid for Community Research 2018 from Ibaraki Prefectural University of Health Sciences.

Conflict of interest

None.

\section{ACKNOWLEDGMENT}

We thank Angela Morben, DVM, ELS, from Edanz Group (www.edanzediting.com/ac), for editing a draft of this manuscript. 


\section{REFERENCES}

Diamond LS, Lynne D, Sigman B: Orthopedic disorders in patients with Down's syndrome. Orthop Clin North Am, 1981, 12: 57-71. [Medline]

Khamis S, Yizhar Z: Effect of feet hyperpronation on pelvic alignment in a standing position. Gait Posture, 2007, 25: 127-134. [Medline] [CrossRef]

3) Tsung BY, Zhang M, Fan YB, et al.: Quantitative comparison of plantar foot shapes under different weight-bearing conditions. J Rehabil Res Dev, 2003, 40: 517-526. [Medline] [CrossRef]

4) Shimizu S, Nagai C, Genda E, et al.: Comparison of foot shapes during gait between low group and normal group of medial longitudinal arch. Supotsu Sangyogaku Kenkyu, 2013, 23: 183-189.

5) Shrout PE, Fleiss JL: Intraclass correlations: uses in assessing rater reliability. Psychol Bull, 1979, 86: 420-428. [Medline] [CrossRef]

6) Saltzman CL, Nawoczenski DA, Talbot KD: Measurement of the medial longitudinal arch. Arch Phys Med Rehabil, 1995, 76: 45-49. [Medline] [CrossRef]

7) Murley GS, Menz HB, Landorf KB: A protocol for classifying normal- and flat-arched foot posture for research studies using clinical and radiographic measurements. J Foot Ankle Res, 2009, 2: 22. [Medline] [CrossRef] 